\title{
Uma abordagem de geração de colunas para o Problema Generalizado de Atribuição
}

\author{
Edson Luiz França Senne (UNESP/FEG) elfsenne@feg.unesp.br \\ Luiz Antonio Nogueira Lorena (INPE/LAC) lorena@inpe.lac.br \\ Silvely Nogueira de Almeida Salomão (UNESP/FCT) silvely@prudente.unesp.br
}

\begin{abstract}
Resumo
A relaxação lagrangeana/surrogate tem sido explorada recentemente como método de estabilização e aceleração de abordagens de geração de colunas. Este trabalho mostra como o uso da relaxação lagrangeana/surrogate conduz para uma abordagem de geração de colunas melhor para o Problema Generalizado de Atribuição (PGA), em comparação com o método de geração de colunas tradicional. O PGA pode ser descrito como o problema de atribuir $n$ tarefas a m máquinas de forma que cada tarefa é atribuída a apenas uma máquina e as capacidades das máquinas são respeitadas. $O$ trabalho apresenta testes computacionais usando problemas disponiveis na literatura para demonstrar a superioridade da abordagem proposta.

Palavras-chave: Relaxação lagrangeana/surrogate, Geração de colunas, Problemas de atribuição.
\end{abstract}

\section{Introdução}

O Problema Generalizado de Atribuição (PGA) é um problema de otimização combinatória NP-difícil (Sahni and Gonzalez, 1976). Sua importância decorre não apenas de sua aplicabilidade direta, mas também do fato de aparecer como um subproblema de muitos outros problemas práticos mais complexos. O problema pode ser estabelecido como o de determinar uma atribuição de custo mínimo de $\mathrm{n}$ tarefas a $\mathrm{m}$ máquinas de forma que cada tarefa é atribuída a apenas uma máquina cuja capacidade deve ser respeitada. Aplicações do PGA aparecem em problemas como roteamento de veículos (Fisher and Jaikumar, 1981), localização de facilidades (Ross and Soland, 1977), escalonamento de recursos (Mazolla et al., 1989), dentre outros. Devido a sua importância, muitos algoritmos heurísticos e exatos já foram propostos para o PGA. Dentre as diversas abordagens de algoritmos heurísticos propostos para o PGA podem ser citadas: particionamento de conjuntos (Cattrysse et al., 1994), busca tabu e simulated annealing (Osman, 1995; Laguna et al., 1995), relaxações lagrangeana e surrogate (Lorena and Narciso, 1996), algoritmo genético (Chu and Beasley, 1997), dentre vários outros. Métodos exatos também têm sido propostos (Savelsberg, 1997; Nauss, 2003).

Definindo $\mathrm{M}=\{1, \ldots, \mathrm{m}\}$ e $\mathrm{N}=\{1, \ldots, \mathrm{n}\}$ e considerando $\mathrm{c}_{\mathrm{ij}}$ como o custo de atribuir a tarefa $\mathrm{j}$ para a máquina $\mathrm{i}, \mathrm{r}_{\mathrm{ij}}$ como a quantidade de recurso requerido para a tarefa $\mathrm{j}$ pela máquina $\mathrm{i}$, $\mathrm{b}_{\mathrm{i}}$ como a capacidade (recursos disponíveis) da máquina $\mathrm{i}$, e a variável de decisão $\mathrm{x}_{\mathrm{ij}}$ definida como: 


$$
\mathrm{x}_{\mathrm{ij}}=\left\{\begin{array}{l}
1, \text { se a tarefa } \mathrm{j} \text { está atribuída para a máquina } \mathrm{i} \\
0, \text { caso contrário }
\end{array}\right.
$$

o PGA pode ser formulado como o seguinte modelo de programação linear inteiro 0-1:

$$
\begin{array}{ll}
\text { Minimizar } \quad & \sum_{\mathrm{i}=1 \mathrm{j}=1}^{\mathrm{m}} \sum_{\mathrm{ij}}^{\mathrm{n}} \mathrm{c}_{\mathrm{ij}} \mathrm{x}_{\mathrm{ij}} \\
\text { sujeito a: } \quad & \sum_{\mathrm{j}=1}^{\mathrm{n}} \mathrm{r}_{\mathrm{ij}} \mathrm{x}_{\mathrm{ij}} \leq \mathrm{b}_{\mathrm{i}}, \quad \forall \mathrm{i} \\
& \sum_{\mathrm{i}=1}^{\mathrm{m}} \mathrm{x}_{\mathrm{ij}}=1, \quad \forall \mathrm{j} \in \mathrm{N} \\
& \mathrm{x}_{\mathrm{ij}} \in\{0,1\}, \quad \forall \mathrm{i} \in \mathrm{M}, \mathrm{j} \in \mathrm{N}
\end{array}
$$

Nessa formulação, a função-objetivo (1) corresponde ao custo total de atribuição, a restrição (2) garante que a capacidade das máquinas é respeitada, a restrição (3) garante que cada tarefa é atribuída a apenas uma máquina, e a restrição (4) impõe que as variáveis sejam binárias.

Para um dado $t \in R$, a relaxação lagrangeana/surrogate do problema $(\mathrm{P})$ pode ser escrita como (Lorena and Senne, 1999):

$$
\begin{array}{ll}
\text { Minimizar } & \sum_{\mathrm{i}=1 \mathrm{j}=1}^{\mathrm{m}} \sum^{\mathrm{n}}\left(\mathrm{c}_{\mathrm{ij}}-\mathrm{t} \lambda_{\mathrm{j}}\right) \mathrm{x}_{\mathrm{ij}}+\sum_{\mathrm{j}=1}^{\mathrm{n}} \lambda_{\mathrm{j}} \\
\text { sujeito a: } & \sum_{\mathrm{j}=1}^{\mathrm{n}} \mathrm{r}_{\mathrm{ij}} \mathrm{x}_{\mathrm{ij}} \leq \mathrm{b}_{\mathrm{i}}, \quad \forall \mathrm{i} \\
& \mathrm{x}_{\mathrm{ij}} \in\{0,1\}, \quad \forall \mathrm{i} \in \mathrm{M}, \mathrm{j} \in \mathrm{N}
\end{array}
$$

Para um dado $\lambda_{j} \in R^{n}$, o melhor valor para o fator $t$ pode ser obtido resolvendo-se $o$ problema dual Max $\mathrm{v}(\mathrm{LSP})$. O interessante a notar é que, se $\mathrm{t}=1$, esta relaxação torna-se a $t \in R$

relaxação lagrangeana usual.

\section{Esquema de Geração de Colunas para o PGA}

Seja $\mathrm{K}_{\mathrm{i}}=\left\{\mathrm{x}_{1}^{\mathrm{i}}, \ldots, \mathrm{x}_{\mathrm{k}_{\mathrm{i}}}^{\mathrm{i}}\right\}$ o conjunto de padrões de atribuição viáveis para a máquina $\mathrm{i}$, onde $\mathrm{x}_{\mathrm{k}}^{\mathrm{i}}=\left\{\mathrm{x}_{1 \mathrm{k}}^{\mathrm{i}}, \ldots, \mathrm{x}_{\mathrm{nk}}^{\mathrm{i}}\right\}$ é uma solução viável para as restrições (2) e (4). Seja $\mathrm{y}_{\mathrm{k}}^{\mathrm{i}}, \mathrm{i} \in \mathrm{M}$ e $\mathrm{k} \in \mathrm{K}_{\mathrm{i}}$, uma variável binária caracterizando se a atribuição $\mathrm{x}_{\mathrm{k}}^{\mathrm{i}}$ está associada realmente à máquina $i$, ou seja:

$$
\mathrm{y}_{\mathrm{k}}^{\mathrm{i}}=\left\{\begin{array}{l}
1, \text { se a atribuição } \mathrm{x}_{\mathrm{k}}^{\mathrm{i}} \text { está associada à máquina } \mathrm{i} \\
0, \text { caso contrário }
\end{array}\right.
$$

Então, o PGA pode ser reformulado como o seguinte problema de cobertura de conjuntos: 
(Q)

$$
\begin{array}{ll}
\text { Minimizar } \quad & \sum_{i=1 \mathrm{k}=1}^{m} \sum_{\mathrm{i}}^{\mathrm{k}_{\mathrm{i}}}\left(\sum_{\mathrm{j}=1}^{\mathrm{n}} \mathrm{c}_{\mathrm{ij}} \mathrm{x}_{\mathrm{jk}}^{\mathrm{i}}\right) \mathrm{y}_{\mathrm{k}}^{\mathrm{i}} \\
\text { sujeito a: } \quad & \sum_{\mathrm{i}=1 \mathrm{k}=1}^{\mathrm{m}} \sum_{\mathrm{k}}^{\mathrm{k}_{\mathrm{i}}} \mathrm{x}_{\mathrm{jk}}^{\mathrm{i}} \mathrm{y}_{\mathrm{k}}^{\mathrm{i}}=1, \quad \mathrm{j} \in \mathrm{N} \\
& \sum_{\mathrm{k}=1}^{\mathrm{k}_{\mathrm{i}}} \mathrm{y}_{\mathrm{k}}^{\mathrm{i}} \leq 1, \quad \mathrm{i} \in \mathrm{M} \\
& \mathrm{y}_{\mathrm{k}}^{\mathrm{i}} \in\{0,1\}, \quad \mathrm{i} \in \mathrm{M}
\end{array}
$$

O problema $(\mathrm{PM})$ a ser resolvido pelo método de geração de colunas é a versão de Programação Linear deste problema de cobertura de conjuntos, ou seja:

$$
\begin{array}{ll}
\text { Minimizar } \quad & \sum_{i=1 \mathrm{k}=1}^{m} \sum_{\mathrm{i}}^{\mathrm{k}_{\mathrm{i}}}\left(\sum_{j=1}^{\mathrm{n}} \mathrm{c}_{\mathrm{ij}} \mathrm{x}_{\mathrm{jk}}^{\mathrm{i}}\right) \mathrm{y}_{\mathrm{k}}^{\mathrm{i}} \\
\text { sujeito a: } \quad & \sum_{\mathrm{i}=1 \mathrm{k}=1}^{\mathrm{m}} \sum_{\mathrm{i}}^{\mathrm{k}_{\mathrm{i}}} \mathrm{x}_{\mathrm{jk}}^{\mathrm{i}} \mathrm{y}_{\mathrm{k}}^{\mathrm{i}}=1, \quad \mathrm{j} \in \mathrm{N} \\
& \sum_{\mathrm{k}=1}^{\mathrm{k}_{\mathrm{i}}} \mathrm{y}_{\mathrm{k}}^{\mathrm{i}} \leq 1, \quad \mathrm{i} \in \mathrm{M} \\
& \mathrm{y}_{\mathrm{k}}^{\mathrm{i}} \in[0,1], \quad \mathrm{i} \in \mathrm{M}
\end{array}
$$

O problema (PM) é conhecido como problema-mestre restrito no contexto do processo de geração de colunas (Barnhart et al., 1998). Na abordagem tradicional, após definir um conjunto inicial de colunas, o problema $(P M)$ é resolvido e os custos duais finais $\pi_{j}(j \in N)$, referentes às restrições $(5)$, e $\mu_{i}(i \in M)$, referentes às restrições (6), são usados para gerar novas colunas. Para isto, as atribuições viáveis $\mathrm{x}_{\mathrm{k}}^{\mathrm{i}}$ para cada máquina $\mathrm{i}$ são obtidas resolvendo-se os seguintes $m$ problemas da mochila:

$$
\begin{array}{ll}
\text { Minimizar } & v\left(M_{i}\right)=\sum_{j=1}^{n}\left(c_{i j}-t \pi j\right) x_{i j} \\
\text { sujeito a: } & \sum_{j=1}^{n} r_{i j} x_{i j} \leq b_{i} \\
& x_{i j} \in\{0,1\}, \quad j \in N
\end{array}
$$

Cada solução viável $\mathrm{x}_{\mathrm{k}}^{\mathrm{i}}$ corresponde a uma coluna na formulação de cobertura de conjuntos. Essa coluna consiste de $\mathrm{n}$ valores iguais a 1 ou 0 representando se a tarefa $\mathrm{j}$ está ou não atribuída à máquina $\mathrm{i}$ (conforme solução do problema da mochila $\mathrm{M}_{\mathrm{i}}$ ) e de um vetor unitário $\overrightarrow{\mathrm{e}_{\mathrm{i}}}$, onde i corresponde ao número da máquina.

Todas as colunas correspondentes a $\mathrm{v}\left(\mathrm{M}_{\mathrm{i}}\right)-\mu_{\mathrm{i}}<0$ são candidatas a serem selecionadas (multi-pricing) e podem ser incorporadas ao problema mestre restrito. É interessante observar 
que como $\mathrm{t} \in \mathrm{R}$ e para cada valor de $\mathrm{t}$ tem-se um novo problema da mochila (e, conseqüentemente, uma nova coluna), com a relaxação lagrangeana/surrogate um número muito maior de colunas, em relação ao enfoque tradicional, que usa $\mathrm{t}=1$, pode ser considerado.

Assim, a combinação da relaxação lagrangena/surrogate com o método de geração de colunas para o PGA pode ser estabelecida pelo seguinte algoritmo:

1. Estabelecer um conjunto inicial de colunas para o problema mestre restrito (PM);

2. Resolver o problema (PM), obtendo os custos duais $\pi_{j}(j \in N)$ e $\mu_{i}(i \in M)$;

3. Resolver os problemas da mochila $\mathrm{M}_{\mathrm{i}}$, considerando valores convenientes $\mathrm{de} \mathrm{t}$ (tipicamente $t \in[0,1]$ ), obtendo as potenciais colunas a serem acrescentadas ao problema mestre restrito;

4. Acrescentar ao problema $(\mathrm{PM})$ todas as colunas correspondentes a $\mathrm{v}\left(\mathrm{M}_{\mathrm{i}}\right)-\mu_{\mathrm{i}}<0(\mathrm{i} \in \mathrm{M})$;

5. Parar, se o passo (4) não acrescentar novas colunas ao problema (PM);

6. Executar os testes de remoção de colunas improdutivas e retornar ao passo (2).

Nos testes computacionais realizados, as colunas improdutivas foram removidas sempre que o problema mestre restrito ultrapassou 5000 colunas. A remoção consistiu em eliminar todas as colunas cujos custos reduzidos fossem maiores do que o custo reduzido médio de todas as colunas do problema.

\section{Testes computacionais}

A abordagem apresentada na seção anterior foi programada na linguagem $\mathrm{C}$ e executada em um microcomputador Pentium III com $1.1 \mathrm{GHz}$ e $512 \mathrm{MB}$ de RAM. Para a solução dos problemas da mochila utilizou-se o algoritmo de Horowitz e Sahni (Martello e Toth, 1990). Foram considerados problemas de Beasley e Yagiura, disponíveis em:

- http://mscmga.ms.ic.ac.uk/jeb/orlib/gapinfo.html (Beasley);

- http://www-or.amp.i.kyoto-u.ac.jp/members/yagiura/gap/ (Yagiura)

para os quais a solução inteira ótima é conhecida. Para efeito de comparação, os problemas foram resolvidos usando-se a relaxação lagrangeana usual (isto é, fixando-se $t=1$ ) e usandose a relaxação lagrangeana/surrogate (para os seguintes valores de $\mathrm{t}=0.5,0.6,0.7,0.8,0.85$, $0.9,0.93,0.95,0.97$ e 1). Os resultados estão mostrados nas tabelas a seguir. Cada uma destas tabelas contém:

- classe - classe do problema

- $\mathrm{m}$ - número de máquinas;

- $\mathrm{n}$ - número de tarefas;

- sol - valor da solução inteira ótima do problema;

- iter - número de iterações;

- cols-a - número de colunas do problema mestre restrito final;

- cols-r - número de colunas removidas do problema mestre restrito;

- LInf - limite inferior para o valor da solução;

- LCpx - limite obtido pelo CPLEX para o problema mestre restrito;

- d\% - desvio percentual do limite obtido pelo CPLEX em relação ao valor da solução inteira ótima, ou seja, $\mathrm{d} \%=100 *|(\mathrm{sol}-\mathrm{LCpx})| /$ sol;

- o tempo computacional (em segundos). 


\begin{tabular}{|c|c|c|c|c|c|c|c|c|c|c|}
\hline classe & $\mathbf{m}$ & $\mathbf{n}$ & sol & iter & cols-a & cols-r & Linf & LCpx & d\% & tempo \\
\hline $\mathrm{A}$ & 5 & 100 & 1698 & 383 & 3575 & 7767 & 1698,00 & 1698,00 & 0,000 & 125,86 \\
\hline & 10 & 100 & 1360 & 68 & 4285 & 2222 & 1360,00 & 1360,00 & 0,000 & 19,33 \\
\hline & 20 & 100 & 1158 & 30 & 5253 & 20 & 1158,00 & 1158,00 & 0,000 & 3,47 \\
\hline & 5 & 200 & 3235 & 2827 & 3332 & 54337 & 3235,00 & 3235,00 & 0,000 & 7217,47 \\
\hline & 10 & 200 & 2623 & 327 & 3732 & 15707 & 2622,96 & 2623,00 & 0,000 & 679,59 \\
\hline & 20 & 200 & 2339 & 93 & 4418 & 10168 & 2339,00 & 2339,00 & 0,000 & 165,35 \\
\hline B & 5 & 100 & 1843 & 176 & 4553 & 12613 & 1838,01 & 1838,84 & 0,226 & 59,45 \\
\hline & 10 & 100 & 1407 & 81 & 4397 & 3975 & 1407,00 & 1407,00 & 0,000 & 18,43 \\
\hline & 20 & 100 & 1166 & 27 & 4132 & 2132 & 1166,00 & 1166,00 & 0,000 & 3,95 \\
\hline & 5 & 200 & 3553 & 1367 & 4268 & 100480 & 3549,01 & 3549,34 & 0,103 & 3524,82 \\
\hline & 10 & 200 & 2831 & 196 & 4840 & 26111 & 2825,03 & 2825,71 & 0,187 & 398,87 \\
\hline & 20 & 200 & 2340 & 95 & 4562 & 13904 & 2338,04 & 2338,57 & 0,061 & 120,85 \\
\hline média & \multicolumn{7}{|c|}{$\mathbf{4 7 3}$} & $\mathbf{4 2 7 9}$ & $\mathbf{2 0 7 8 6}$ & \\
\hline
\end{tabular}

Tabela 1. Resultados da relaxação lagrangeana/surrogate (problemas pequenos - Beasley)

\begin{tabular}{|c|c|c|c|c|c|c|c|c|c|r|}
\hline classe & $\mathbf{m}$ & $\mathbf{n}$ & sol & iter & cols-a & cols-r & Linf & LCpx & d\% & tempo \\
\hline $\mathrm{A}$ & 5 & 100 & 1698 & 897 & 4959 & 2162 & 1697,04 & 1698,00 & 0,000 & 228,25 \\
\hline & 10 & 100 & 1360 & 176 & 4178 & 0 & 1360,00 & 1360,00 & 0,000 & 30,21 \\
\hline & 20 & 100 & 1158 & 54 & 3193 & 0 & 1158,00 & 1158,00 & 0,000 & 5,02 \\
\hline & 5 & 200 & 3235 & 4478 & 4726 & 25216 & 3234,51 & 3235,00 & 0,000 & 10715,07 \\
\hline & 10 & 200 & 2623 & 972 & 4028 & 9116 & 2623,00 & 2623,00 & 0,000 & 1580,23 \\
\hline & 20 & 200 & 2339 & 214 & 3791 & 3956 & 2338,25 & 2339,00 & 0,000 & 307,22 \\
\hline B & 5 & 100 & 1843 & 496 & 4470 & 4064 & 1838,03 & 1838,84 & 0,226 & 192,69 \\
\hline & 10 & 100 & 1407 & 135 & 4074 & 0 & 1407,00 & 1407,00 & 0,000 & 19,58 \\
\hline & 20 & 100 & 1166 & 44 & 3157 & 0 & 1166,00 & 1166,00 & 0,000 & 5,36 \\
\hline & 5 & 200 & 3553 & 2314 & 3409 & 35220 & 3549,02 & 3549,53 & 0,098 & 5549,72 \\
\hline & 10 & 200 & 2831 & 431 & 4991 & 7949 & 2825,12 & 2825,51 & 0,194 & 900,12 \\
\hline & 20 & 200 & 2340 & 156 & 4819 & 2480 & 2338,10 & 2338,52 & 0,063 & 219,68 \\
\hline média & & & & $\mathbf{8 6 4}$ & $\mathbf{4 1 5 0}$ & $\mathbf{7 5 1 4}$ & & & $\mathbf{0 , 0 4 8}$ & $\mathbf{1 6 4 6 , 1 0}$ \\
\hline
\end{tabular}

Tabela 2. Resultados da relaxação lagrangeana (problemas pequenos - Beasley)

\begin{tabular}{|c|c|c|c|c|c|c|c|c|c|c|}
\hline classe & $\mathbf{m}$ & $\mathbf{n}$ & $\mathbf{s o l}$ & $\mathbf{i t e r}$ & $\mathbf{c o l s - a}$ & cols-r & LInf & LCpx & $\mathbf{d \%}$ & tempo \\
\hline $\mathrm{C}$ & 5 & 100 & 1931 & 185 & 3361 & 12585 & 1929,06 & 1929,67 & 0,069 & 61,05 \\
\hline & 10 & 100 & 1402 & 53 & 4957 & 4246 & 1399,58 & 1399,89 & 0,150 & 7,75 \\
\hline & 20 & 100 & 1243 & 21 & 4081 & 2373 & 1241,50 & 1241,67 & 0,107 & 2,33 \\
\hline & 5 & 200 & 3456 & 1456 & 4373 & 107906 & 3454,16 & 3454,56 & 0,042 & 3282,77 \\
\hline & 10 & 200 & 2806 & 183 & 3280 & 25970 & 2803,05 & 2803,95 & 0,073 & 331,14 \\
\hline & 20 & 200 & 2391 & 67 & 4835 & 13948 & 2390,05 & 2390,17 & 0,035 & 96,49 \\
\hline $\mathrm{D}$ & 5 & 100 & 6353 & 192 & 4784 & 6542 & 6349,08 & 6349,93 & 0,048 & 64,80 \\
\hline & 10 & 100 & 6349 & 42 & 4088 & 2231 & 6341,17 & 6341,45 & 0,119 & 10,98 \\
\hline & 20 & 100 & 6196 & 18 & 5442 & 20 & 6176,09 & 6176,14 & 0,321 & 3,23 \\
\hline & 5 & 200 & 12743 & 1228 & 3490 & 49561 & 12740,04 & 12740,04 & 0,023 & 4449,99 \\
\hline$*$ & 10 & 200 & 12433 & 221 & 4627 & 17475 & 12425,03 & 12425,61 & 0,059 & 495,88 \\
\hline E & 20 & 200 & 12244 & 58 & 3607 & 12662 & 12229,11 & 12229,70 & 0,117 & 122,59 \\
\hline & 5 & 100 & 12681 & 191 & 4619 & 7961 & 12673,05 & 12673,05 & 0,063 & 66,88 \\
\hline & 10 & 100 & 11577 & 71 & 3722 & 5447 & 11568,01 & 11568,02 & 0,078 & 12,69 \\
\hline & 20 & 100 & 8436 & 25 & 3482 & 5374 & 8431,27 & 8431,52 & 0,053 & 4,76 \\
\hline & 5 & 200 & 24930 & 1626 & 3813 & 70077 & 24926,03 & 24926,69 & 0,013 & 4086,47 \\
\hline & 10 & 200 & 23307 & 369 & 3820 & 25744 & 23302,02 & 23302,05 & 0,021 & 646,15 \\
\hline média & 20 & 200 & 22379 & 93 & 3673 & 20471 & 22376,03 & 22376,79 & 0,010 & 160,75 \\
\hline
\end{tabular}

Tabela 3. Resultados da relaxação lagrangeana/surrogate (problemas grandes - Beasley) 


\begin{tabular}{|c|c|c|c|c|c|c|c|c|c|c|}
\hline classe & $\mathbf{m}$ & $\mathbf{n}$ & sol & iter & cols-a & cols-r & LInf & LCpx & d\% & cpu \\
\hline $\mathrm{C}$ & 5 & 100 & 1931 & 404 & 3354 & 3990 & 1929,03 & 1929,83 & 0,061 & 115,72 \\
\hline & 10 & 100 & 1402 & 103 & 4067 & 0 & 1399,43 & 1399,86 & 0,153 & 12,28 \\
\hline & 20 & 100 & 1243 & 34 & 3094 & 0 & 1241,20 & 1241,80 & 0,097 & 2,64 \\
\hline & 5 & 200 & 3456 & 2432 & 4517 & 33566 & 3454,03 & 3454,49 & 0,044 & 6055,82 \\
\hline & 10 & 200 & 2806 & 424 & 4743 & 8021 & 2803,05 & 2803,95 & 0,073 & 952,11 \\
\hline & 20 & 200 & 2391 & 144 & 3771 & 3774 & 2390,09 & 2390,17 & 0,035 & 180,57 \\
\hline $\mathrm{D}$ & 5 & 100 & 6353 & 301 & 3915 & 3916 & 6349,07 & 6349,92 & 0,048 & 78,43 \\
\hline & 10 & 100 & 6349 & 66 & 4097 & 0 & 6341,39 & 6341,45 & 0,119 & 10,86 \\
\hline & 20 & 100 & 6196 & 31 & 3571 & 0 & 6176,14 & 6176,14 & 0,321 & 3,74 \\
\hline & 5 & 200 & 12743 & 1343 & 3719 & 27243 & 12740,02 & 12740,04 & 0,023 & 3495,80 \\
\hline & 10 & 200 & 12433 & 284 & 3470 & 10152 & 12425,05 & 12425,73 & 0,058 & 584,47 \\
\hline & 20 & 200 & 12244 & 93 & 4160 & 4154 & 12229,06 & 12229,69 & 0,117 & 156,01 \\
\hline & 5 & 100 & 12681 & 333 & 4294 & 1921 & 12673,05 & 12673,05 & 0,063 & 79,60 \\
\hline & 10 & 100 & 11577 & 102 & 4239 & 0 & 11568,02 & 11568,02 & 0,078 & 15,15 \\
\hline & 20 & 100 & 8436 & 39 & 3601 & 0 & 8431,10 & 8431,53 & 0,053 & 5,58 \\
\hline & 5 & 200 & 24930 & 2531 & 4653 & 31003 & 24926,07 & 24926,64 & 0,013 & 5703,46 \\
\hline & 10 & 200 & 23307 & 499 & 4380 & 9005 & 23302,05 & 23302,05 & 0,021 & 772,70 \\
\hline & 20 & 200 & 22379 & 150 & 3776 & 4823 & 22376,16 & 22376,79 & 0,010 & 219,32 \\
\hline média & & & & $\mathbf{5 1 7}$ & $\mathbf{3 9 6 8}$ & $\mathbf{7 8 6 5}$ & & & $\mathbf{0 , 0 7 7}$ & $\mathbf{1 0 2 4 , 6 8}$ \\
\hline
\end{tabular}

Tabela 4. Resultados da relaxação lagrangeana (problemas grandes - Beasley)

\begin{tabular}{|c|c|c|c|c|c|c|c|c|c|c|}
\hline classe & $\mathbf{m}$ & $\mathbf{n}$ & sol & iter & cols-a & cols-r & Linf & LCpx & d\% & tempo \\
\hline $\mathrm{C}$ & 20 & 400 & 4781 & 408 & 4629 & 90997 & 4780,00 & 4780,18 & 0,017 & 4898,72 \\
\hline & 40 & 400 & 4244 & 104 & 4298 & 52408 & 4243,10 & 4243,45 & 0,013 & 1506,70 \\
\hline $\mathrm{D}$ & 20 & 400 & 24561 & 268 & 5077 & 59420 & 24560,03 & 24560,23 & 0,003 & 6730,81 \\
\hline & 40 & 400 & 24350 & 84 & 4050 & 59569 & 24349,04 & 24349,50 & 0,002 & 1871,64 \\
\hline $\mathrm{E}$ & 20 & 400 & 44876 & 489 & 3921 & 99763 & 44875,03 & 44875,48 & 0,001 & 9075,33 \\
\hline & 40 & 400 & 44557 & 149 & 3945 & 82365 & 44556,01 & 44556,88 & 0,000 & 2722,58 \\
\hline média & & & & $\mathbf{2 5 0}$ & $\mathbf{4 3 2 0}$ & $\mathbf{7 4 0 8 7}$ & $\mathbf{2 4 5 6 0 , 5 4}$ & $\mathbf{2 4 5 6 0 , 9 5}$ & $\mathbf{0 , 0 0 6}$ & $\mathbf{4 4 6 7 , 6 3}$ \\
\hline
\end{tabular}

Tabela 5. Resultados da relaxação lagrangeana/surrogate (problemas grandes - Yagiura)

\begin{tabular}{|c|c|c|c|c|c|c|c|c|c|c|}
\hline classe & $\mathbf{m}$ & $\mathbf{n}$ & sol & iter & Cols-a & cols-r & LInf & LCpx & d\% & tempo \\
\hline $\mathrm{C}$ & 20 & 400 & 4781 & 602 & 3969 & 24671 & 4780,01 & 4780,19 & 0,017 & 7231,76 \\
\hline & 40 & 400 & 4244 & 162 & 3468 & 11486 & 4243,04 & 4243,45 & 0,013 & 1994,91 \\
\hline $\mathrm{D}$ & 20 & 400 & 24561 & 345 & 3625 & 26212 & 24560,10 & 24560,21 & 0,003 & 6906,88 \\
\hline & 40 & 400 & 24350 & 149 & 4769 & 17795 & 24349,42 & 24349,50 & 0,002 & 2628,16 \\
\hline $\mathrm{E}$ & 20 & 400 & 44876 & 647 & 3572 & 29223 & 44875,13 & 44875,49 & 0,001 & 7466,17 \\
\hline & 40 & 400 & 44557 & 235 & 4948 & 17015 & 44556,38 & 44556,88 & 0,000 & 3358,14 \\
\hline média & & & & $\mathbf{3 5 7}$ & $\mathbf{4 0 5 9}$ & $\mathbf{2 1 0 6 7}$ & $\mathbf{2 4 5 6 0 , 6 8}$ & $\mathbf{2 4 5 6 0 , 9 5}$ & $\mathbf{0 , 0 0 6}$ & $\mathbf{4 9 3 1 , 0 0}$ \\
\hline
\end{tabular}

Tabela 6. Resultados da relaxação lagrangeana (problemas grandes - Yagiura)

As tabelas a seguir apresentam as relações de tempo de processamento para os resultados obtidos pela relaxação lagrangeana/surrogate e pela relaxação lagrangeana. Nas tabelas a seguir, a coluna S/L representa a razão entre os tempos obtidos pela relaxação lagrangeana/surrogate e os tempos obtidos pela relaxação lagrangeana para cada um dos problemas. 


\begin{tabular}{|c|c|c|c|}
\hline classe & $\mathbf{m}$ & $\mathbf{n}$ & $\mathbf{S} / \mathbf{L}$ \\
\hline $\mathrm{A}$ & 5 & 100 & 0,55 \\
\hline & 10 & 100 & 0,64 \\
\hline & 20 & 100 & 0,69 \\
\hline & 5 & 200 & 0,67 \\
\hline & 10 & 200 & 0,43 \\
\hline & 20 & 200 & 0,54 \\
\hline $\mathrm{B}$ & 5 & 100 & 0,31 \\
\hline & 10 & 100 & 0,94 \\
\hline & 20 & 100 & 0,74 \\
\hline & 5 & 200 & 0,64 \\
\hline & 10 & 200 & 0,44 \\
\hline & 20 & 200 & 0,55 \\
\hline média & \multicolumn{3}{|c}{$\mathbf{0 , 5 9}$} \\
\hline
\end{tabular}

Tabela 7. Relação de tempos (problemas pequenos - Beasley)

\begin{tabular}{|c|c|c|c|}
\hline classe & $\mathbf{m}$ & $\mathbf{n}$ & $\mathbf{S} / \mathbf{L}$ \\
\hline $\mathrm{C}$ & 5 & 100 & 0,53 \\
\hline & 10 & 100 & 0,63 \\
\hline & 20 & 100 & 0,88 \\
\hline & 5 & 200 & 0,54 \\
\hline & 10 & 200 & 0,35 \\
\hline & 20 & 200 & 0,53 \\
\hline $\mathrm{D}$ & 5 & 100 & 0,83 \\
\hline & 10 & 100 & 1,01 \\
\hline & 20 & 100 & 0,86 \\
\hline & 5 & 200 & 1,27 \\
\hline & 10 & 200 & 0,85 \\
\hline & 20 & 200 & 0,79 \\
\hline $\mathrm{E}$ & 5 & 100 & 0,84 \\
\hline & 10 & 100 & 0,84 \\
\hline & 20 & 100 & 0,85 \\
\hline & 5 & 200 & 0,72 \\
\hline & 10 & 200 & 0,84 \\
\hline & 20 & 200 & 0,73 \\
\hline média & & & $\mathbf{0 , 7 7}$ \\
\hline
\end{tabular}

Tabela 8. Relação de tempos (problemas grandes - Beasley)

\begin{tabular}{|c|c|c|c|}
\hline classe & $\mathbf{m}$ & $\mathbf{n}$ & $\mathbf{S} / \mathbf{L}$ \\
\hline $\mathrm{C}$ & 20 & 400 & 0,68 \\
\hline & 40 & 400 & 0,76 \\
\hline $\mathrm{D}$ & 20 & 400 & 0,97 \\
\hline & 40 & 400 & 0,71 \\
\hline $\mathrm{E}$ & 20 & 400 & 1,22 \\
\hline & 40 & 400 & 0,81 \\
\hline Media & & & $\mathbf{0 , 8 6}$ \\
\hline
\end{tabular}

Tabela 9. Relação de tempos (problemas grandes - Yagiura) 


\section{Conclusão}

A relaxação lagrangeana/surrogate tem sido explorada como uma alternativa computacional mais rápida do que a relaxação lagrangeana tradicional para a solução de diversos problemas de otimização combinatória, em especial para abordagens de geração de colunas (Lorena and Senne, 2004).

Como se nota pelos resultados computacionais apresentados neste trabalho, a abordagem de geração de colunas pode se beneficiar com o emprego da relaxação lagrangeana/surrogate, produzindo mais colunas para o problema mestre restrito e obtendo bons resultados em menor tempo computacional.

\section{Referências bibliográficas}

Sahni, S.; Gonzalez, T. (1976) P-complete approximation problems. J. ACM, 23, 555-565.

Cattrysse, D.G.; Salomon, M.; Van Wassenhove, L.N. (1994). A set partitioning heuristic for the generalized assignment problem. European J. Oper. Res. 72 167-174.

Chu, P.C.; Beasley, J.E. (1997). A genetic algorithm for the generalized assignment problem. Computers Oper. Res. 24 17-23.

Osman, I.H. (1995). Heuristics for the generalized assignment problem: simulated annealing and tabu search approaches. OR Spektrum, 17, 211-225.

Laguna, M., Kelly, J.P.; Gonzalez-Velarde, J.L.; Glover, F. (1995). Tabu search for the multilevel generalized assignment problem. European J. Oper. Res. 82 176-189.

Lorena, L.A.N.; Narciso, M.G. (1996). Relaxation heuristics for a generalized assignment problem. European J. Oper. Res. 91 600-610.

Savelsbergh, M. (1997). A branch-and-price algorithm for the generalized assignment problem. Oper. Res. 45 831-841.

Nauss, R.M. (2003). Solving the generalized assignment problem: an optimizing and heuristic approach. INFORMS Journal on Computing, 15 (3), 249-266.

Fisher, M.; Jaikumar, R. (1981).A generalized assignment heuristic for vehicle routing. Networks, 11, 109-124.

Ross, G.T.; Soland, R.M. (1977).Modeling facility location problems as generalized assignment problems. Management Sci., 24, 345-357.

Mazzola, J.; Neebe, A.; Dunn, C. (1989).Production planning of flexible manufacturing system in material requirements planning environment. Internat. J. Flexible Manufacturing Systems, 1/2, 115-142.

Lorena, L.A.N.; Senne, E.L.F. (1999) Improving Traditional Subgradient Scheme for Lagrangean Relaxation: An Application to Location Problems. International Journal on Mathematical Algorithms, 1, 133-151.

Martello,S.; Toth, P. (1990) Knapsack problems: algorithms and computer implementations. New York, Wiley.

Lorena, L.A.N.; Senne, E.L.F. (2004) A Column Generation Approach to Capacitated pMedian Problems, Computers \& Operations Research, 31 (6): 863-876, 2004. 